\title{
Boundedness of Oscillatory Integrals with Variable Calderón-Zygmund Kernel on Weighted Morrey Spaces
}

\author{
Yali Pan, ${ }^{1}$ Changwen Li, ${ }^{1}$ and Xinsong Wang ${ }^{2}$ \\ ${ }^{1}$ School of Mathematical Sciences, Huaibei Normal University, Huaibei, Anhui 235000, China \\ ${ }^{2}$ School of Science, Tianjin Chengjian University, Tianjin 300384, China \\ Correspondence should be addressed to Changwen Li; cwli2008@163.com
}

Received 17 August 2013; Accepted 11 October 2013

Academic Editor: Yoshihiro Sawano

Copyright (c) 2013 Yali Pan et al. This is an open access article distributed under the Creative Commons Attribution License, which permits unrestricted use, distribution, and reproduction in any medium, provided the original work is properly cited.

Oscillatory integral operators play a key role in harmonic analysis. In this paper, the authors investigate the boundedness of the oscillatory singular integrals with variable Calderón-Zygmund kernel on the weighted Morrey spaces $L^{p, k}(\omega)$. Meanwhile, the corresponding results for the oscillatory singular integrals with standard Calderón-Zygmund kernel are established.

\section{Introduction and Main Results}

Suppose that $k$ is the standard Calderón-Zygmund kernel. That is, $k \in C^{\infty}\left(\mathbb{R}^{n} \backslash\{0\}\right)$ is homogeneous of degree $n$, and $\int_{\Sigma} k(x) d \sigma_{x}=0$, where $\Sigma=\left\{x \in \mathbb{R}^{n}:|x|=1\right\}$. The oscillatory integral operator $T_{\lambda}$ is defined by

$$
T_{\lambda} f(x)=p \cdot v \cdot \int_{\mathbb{R}^{n}} e^{i \lambda \Phi(x, y)} k(x-y) \varphi(x, y) f(y) d y,
$$

where $\lambda \in \mathbb{R}, \varphi \in C_{0}^{\infty}\left(\mathbb{R}^{n} \times \mathbb{R}^{n}\right)$, where $C_{0}^{\infty}\left(\mathbb{R}^{n} \times \mathbb{R}^{n}\right)$ is the space of infinitely differentiable functions on $\mathbb{R}^{n} \times \mathbb{R}^{n}$ with compact supports, and $\Phi$ is a real-analytic function or a real- $C^{\infty}\left(\mathbb{R}^{n} \times \mathbb{R}^{n}\right)$ function satisfying that, for any $\left(x_{0}, y_{0}\right) \in$ $\operatorname{supp} \varphi$, there exists $\left(j_{0}, k_{0}\right), 1 \leq j_{0}, k_{0} \leq n$, such that $\partial^{2} \Phi\left(x_{0}, y_{0}\right) / \partial x_{j_{0}} \partial y_{k_{0}}$ does not vanish up to infinite order. These operators have arisen in the study of singular integrals supported on lower dimensional varieties and the singular Radon transform. In [1], Pan proved that $T_{\lambda}$ are uniform in $\lambda$ bounded on $L^{p}\left(\mathbb{R}^{n}\right)(1<p<\infty)$. Lu et al. [2] proved the weighted $L^{p}$ boundedness of $T_{\lambda}$ defined by (1).

Let $k(x, y)$ be a variable Calderón-Zygmund kernel. That means, for a.e. $x \in \mathbb{R}^{n}, k(x, \cdot)$ is a standard CalderónZygmund kernel and

$$
\max _{|j| \leq 2 n, j \in \mathbb{Z}}\left\|\frac{\partial^{|j|} k}{\partial y^{j}}\right\|_{L^{\infty}\left(\mathbb{R}^{n} \times \Sigma\right)}=A<\infty .
$$

Define the oscillatory integral operator with variable Calderón-Zygmund kernel $T_{\lambda}^{*}$ by

$$
T_{\lambda}^{*} f(x)=p \cdot v \cdot \int_{\mathbb{R}^{n}} e^{i \lambda \Phi(x, y)} k(x, x-y) \varphi(x, y) f(y) d y,
$$

where $\lambda, \varphi$, and $\Phi$ satisfy the same assumptions as those in the operator defined by (1).

Lu et al. [2] investigated the $L^{p}$ and weighted $L^{p}$ boundedness about this class of oscillatory integral operators.

The classical Morrey space $L^{p, \lambda}$ was first introduced by Morrey in [3] to study the local behavior of solutions to second order elliptic partial differential equations. In 2009, Komori and Shirai [4] first defined the weighted Morrey spaces $L^{p, \kappa}(\omega)$ which could be viewed as an extension of weighted Lebesgue spaces. They studied the boundedness of the fractional integral operator, the Hardy-Littlewood maximal operator, and the Calderón-Zygmund singular integral operator on the space. The boundedness results about some operators on these spaces can be see in ([5-17]). Recently, Shi et al. [18] obtained the boundedness of a class of oscillatory integrals with Calderón-Zygmund kernel and polynomial phase on weighted Morrey spaces. Their results are stated as follows. 
Let $P(x, y)$ be a real valued polynomial defined on $\mathbb{R}^{n} \times$ $\mathbb{R}^{n}$ and let $k$ satisfy the following hypotheses:

$$
\begin{gathered}
|k(x, y)| \leq \frac{C}{|x-y|^{n}}, \quad \forall x \neq y, \\
\left|\nabla_{x} k(x, y)\right|+\left|\nabla_{y} k(x, y)\right| \leq \frac{C}{|x-y|^{n+1}}, \quad \forall x \neq y .
\end{gathered}
$$

We define

$$
\begin{gathered}
S f(x)=p \cdot v \cdot \int_{\mathbb{R}^{n}} k(x, y) f(y) d y, \\
R f(x)=p \cdot v \cdot \int_{\mathbb{R}^{n}} e^{i P(x, y)} k(x, y) f(y) d y .
\end{gathered}
$$

Theorem A (see [18]). Let $1<p<\infty, 0<\kappa<1$, and $\omega \in A_{p}$. If $S$ is of type $\left(L^{2}, L^{2}\right)$, then, for any real polynomial $P(x, y)$, there exists a constant $C>0$ such that

$$
\|R f\|_{L^{p, \kappa}(\omega)} \leq C\|f\|_{L^{p, \kappa}(\omega)}
$$

The purpose of this paper is to generalize the above results to the case with real- $C^{\infty}$ or analytic phase functions. Our main results in this paper are formulated as follows.

Theorem 1. Let $\lambda \in \mathbb{R}, \varphi \in C_{0}^{\infty}\left(\mathbb{R}^{n} \times \mathbb{R}^{n}\right)$, and $\Phi$ a real$C^{\infty}\left(\mathbb{R}^{n} \times \mathbb{R}^{n}\right)$ function satisfying that, for any $\left(x_{0}, y_{0}\right) \in$ $\operatorname{supp} \varphi$, there exists $\left(j_{0}, k_{0}\right), 1 \leq j_{0}, k_{0} \leq n$, such that $\partial^{2} \Phi\left(x_{0}, y_{0}\right) / \partial x_{j_{0}} \partial y_{k_{0}}$ does not vanish up to infinite order. Assume that $k$ is a standard Calderón-Zygmund kernel and $T_{\lambda}$ is defined as in (1). Then for any $1<p<\infty, 0<\kappa<1$, and $\omega \in A_{p}, T_{\lambda}$ is bounded on $L^{p, \kappa}(\omega)$.

Theorem 2. Let $\lambda \in \mathbb{R}, \varphi \in C_{0}^{\infty}\left(\mathbb{R}^{n} \times \mathbb{R}^{n}\right)$, and $\Phi$ a real$C^{\infty}\left(\mathbb{R}^{n} \times \mathbb{R}^{n}\right)$ function satisfying that, for any $\left(x_{0}, y_{0}\right) \in$ $\operatorname{supp} \varphi$, there exists $\left(j_{0}, k_{0}\right), 1 \leq j_{0}, k_{0} \leq n$, such that $\partial^{2} \Phi\left(x_{0}, y_{0}\right) / \partial x_{j_{0}} \partial y_{k_{0}}$ does not vanish up to infinite order. Assume that $k$ is a variable Calderón-Zygmund kernel and $T_{\lambda}^{*}$ is defined as in (3). Then for any $1<p<\infty, 0<\kappa<1$, and $\omega \in A_{p}, T_{\lambda}^{*}$ is bounded on $L^{p, \kappa}(\omega)$.

\section{Notations and Preliminary Lemmas}

Let $B=B\left(x_{0}, r\right)$ be the ball with the center $x_{0}$ and radius $r$. Given a ball $B$ and $\lambda>0, \lambda B$ denotes the ball with the same center as $B$ whose radius is $\lambda$ times that of $B$.

The classical $A_{p}$ weighted theory was first introduced by Muckenhoupt in [19]. A weight $\omega$ is a locally integrable function on $\mathbb{R}^{n}$, which takes values in $(0, \infty)$ a.e. For a given weight function $\omega$, we denote the Lebesgue measure of $B$ by $|B|$ and the weighted measure of $E$ by $\omega(E)$; that is, $\omega(E)=$ $\int_{E} \omega(x) d x$. Given a weight $\omega$, we say that $\omega$ satisfies the doubling condition if there exists a constant $D>0$ such that, for any ball $B$, we have $\omega(2 B) \leq D \omega(B)$.

We say $\omega \in A_{p}$ with $1<p<\infty$, if there exists a constant $C>0$, such that

$$
\left(\frac{1}{|B|} \int_{B} \omega(x) d x\right)\left(\frac{1}{|B|} \int_{B} \omega(x)^{-1 /(p-1)} d x\right)^{p-1} \leq C
$$

for every ball $B \subseteq \mathbb{R}^{n}$. When $p=1, \omega \in A_{1}$ if there exists $C>0$, such that

$$
\frac{1}{|B|} \int_{B} \omega(x) d x \leq C \text { ess } \inf _{x \in B} \omega(x),
$$

for almost every $x \in \mathbb{R}^{n}$. We define $A_{\infty}=\bigcup_{p \geq 1} A_{p}$. A weight function $\omega$ is said to belong to the reverse Hölder class $R H_{r}$ if there exist two constants $r>0$ and $C>0$ such that the following reverse Hölder inequality holds:

$$
\left(\frac{1}{|B|} \int_{B} \omega(x)^{r} d x\right)^{1 / r} \leq C\left(\frac{1}{|B|} \int_{B} \omega(x) d x\right),
$$

for every ball $B \subseteq \mathbb{R}^{n}$.

It is well known that, if $\omega \in A_{p}$ with $1 \leq p<\infty$, then there exists $r>1$ such that $\omega \in R H_{r}$.

Lemma 3 (see [20]). Let $\omega \in A_{p}, p \geq 1$, and $r>0$. Then for any ball $B$ and $\lambda>1$,

$$
\begin{gathered}
\omega(2 B) \leq C \omega(B), \\
\omega(\lambda B) \leq C \lambda^{n p} \omega(B),
\end{gathered}
$$

where $C$ does not depend on $B$ nor on $\lambda$.

Lemma 4 (see [21]). Let $\omega \in R H_{r}$ with $r>1$. Then there exists a constant $C$ such that

$$
\frac{\omega(E)}{\omega(B)} \leq C\left(\frac{|E|}{|B|}\right)^{(r-1) / r},
$$

for any measurable subset $E$ of a ball B.

The weighted Morrey spaces were defined as follows.

Definition 5 (see [4]). Let $1 \leq p<\infty, 0<\kappa<1$, and $\omega$ a weight function. Then the weighted Morrey space is defined by

$$
L^{p, \kappa}(\omega)=\left\{f \in L_{\mathrm{loc}}^{p}(\omega):\|f\|_{L^{p, \kappa}(\omega)}<\infty\right\}
$$

where

$$
\|f\|_{L^{p, \kappa}(\omega)}=\sup _{B}\left(\frac{1}{\omega(B)^{\kappa}} \int_{B}|f(x)|^{p} \omega(x) d x\right)^{1 / p},
$$

and the supremum is taken over all balls $B$ in $\mathbb{R}^{n}$. The space $L_{\text {loc }}^{p}(\omega)$ is defined by

$$
\begin{aligned}
& L_{\mathrm{loc}}^{p}(\omega)=\left\{f: f \chi_{K} \in L^{p}(\omega),\right. \\
&\text { for every compact set } \left.K \subseteq \mathbb{R}^{n}\right\} .
\end{aligned}
$$

Our argument is based heavily on the following results.

Lemma 6 (see [2]). Assume that $T_{\lambda}$ is defined as in (1). Then for any $1<p<\infty$ and $\omega \in A_{p}$, one has

$$
\left\|T_{\lambda} f\right\|_{L^{p}(\omega)} \leq C\left(n, p, \Phi, \varphi, C_{p, \omega}\right) B\|f\|_{L^{p}(\omega)},
$$

where $C\left(n, p, \Phi, \varphi, C_{p, \omega}\right)$ is independent of $\lambda, k$, and $f$ and $B=$ $\|k\|_{C^{1}(\Sigma)}$. 
Lemma 7 (see [2]). Assume that $T_{\lambda}^{*}$ is defined as in (3). Then for any $1<p<\infty$ and $\omega \in A_{p}$, one has

$$
\left\|T_{\lambda}^{*} f\right\|_{L^{p}(\omega)} \leq C\left(n, p, \Phi, \varphi, C_{p, \omega}\right) A\|f\|_{L^{p}(\omega)},
$$

where $C\left(n, p, \Phi, \varphi, C_{p, \omega}\right)$ is independent of $\lambda, k$, and $f . A$ is defined in (2).

Definition 8 (see [4]). The Hardy-Littlewood maximal operator $M$ is defined by

$$
M f(x)=\sup _{B \ni x} \frac{1}{|B|} \int_{B}|f(y)| d y, \quad f \in L_{\mathrm{loc}}\left(\mathbb{R}^{n}\right) .
$$

Lemma 9 (see [4]). If $1<p<\infty, 0<\kappa<1$, and $\omega \in A_{p}$ then the Hardy-Littlewood maximal operator $M$ is bounded on $L^{p, \kappa}(\omega)$.

Lemma 10 (see [22]). Denote by $\mathscr{H}_{m}$ the spaces of spherical harmonic functions of degree $m$. Then

(a) $L^{2}(\Sigma)=\oplus_{m=0}^{\infty} \mathscr{H}_{m}$, and $g_{m}=\operatorname{dim} \mathscr{H}_{m} \leq C(n) m^{n-2}$ for any $m \in \mathbb{N}$;

(b) for any $m=0,1,2, \ldots$, there exists an orthogonal system $\left\{Y_{j m}\right\}_{j=1}^{g_{m}}$ of $\mathscr{H}_{m}$ such that $\left\|Y_{j m}\right\|_{L^{\infty}(\Sigma)} \leq$ $C(n) m^{n / 2-1}, Y_{j m}=(-m)^{-n}(m+n-2)^{-n} \Lambda^{n} Y_{j m}, j=$ $1, \ldots, g_{m}$, and $\Lambda$ is the Beltrami-Laplace operator on $\Sigma$.

In the following the letter $C$ will denote a constant which may vary at each occurrence.

\section{Proof of Theorems}

Proof of Theorem 1. It is sufficient to prove that there exists a constant $C>0$ such that

$$
\frac{1}{\omega(B)^{\kappa}} \int_{B}\left|T_{\lambda} f(x)\right|^{p} \omega(x) d x \leq C\|f\|_{L^{p, \kappa}(\omega)}^{p} .
$$

Fix a ball $B=B\left(x_{0}, r_{B}\right)$ and decompose $f=f_{1}+f_{2}$, with $f_{1}=f \chi_{2 B}$. Then we have

$$
\begin{aligned}
& \frac{1}{\omega(B)^{\kappa}} \int_{B}\left|T_{\lambda} f(x)\right|^{p} \omega(x) d x \\
& \leq C\left\{\frac{1}{\omega(B)^{\kappa}} \int_{B}\left|T_{\lambda} f_{1}(x)\right|^{p} \omega(x) d x\right. \\
& \left.\quad+\frac{1}{\omega(B)^{\kappa}} \int_{B}\left|T_{\lambda} f_{2}(x)\right|^{p} \omega(x) d x\right\} \\
& =C\left\{I_{1}+I_{2}\right\} .
\end{aligned}
$$

Using Lemmas 3 and 6, we get

$$
\begin{aligned}
I_{1} & \leq C \frac{1}{\omega(B)^{\kappa}} \int_{2 B}|f(x)|^{p} \omega(x) d x \\
& \leq C\|f\|_{L^{p, \kappa}(\omega)}^{p} \cdot \frac{\omega(2 B)^{\kappa}}{\omega(B)^{\kappa}} \\
& \leq C\|f\|_{L^{p, \kappa}(\omega)}^{p} .
\end{aligned}
$$

We now estimate $I_{2}$. We can write

$$
\left|T_{\lambda} f_{2}(x)\right|=\left|\int_{(2 B)^{c}} e^{i \lambda \Phi(x, y)} k(x-y) \varphi(x, y) f(y) d y\right| .
$$

Now by an argument similar to the proof of Lemma 6 in [2], we choose $\phi_{1} \in C_{0}^{\infty}\left(\mathbb{R}^{n}\right)$ such that $\phi_{1}(x) \equiv 1$, when $|x| \leq$ 1 , and $\phi_{1}(x) \equiv 0$ when $|x|>2$. Let $\phi_{2}=1-\phi_{1}$ and $N \in \mathbb{N}$ which is large enough and will be determined later. Write

$$
k(x)=k_{\lambda}^{1}(x)+k_{\lambda}^{2}(x),
$$

where

$$
k_{\lambda}^{j}(x)=k(x) \phi_{j}\left(\lambda^{1 / N} x\right), \quad j=1,2 .
$$

Then

$$
\begin{aligned}
T_{\lambda} f_{2}(x)= & p \cdot v \cdot \int_{(2 B)^{c}} e^{i \lambda \Phi(x, y)} k_{\lambda}^{1}(x-y) \\
& \times \varphi(x, y) f(y) d y \\
& +p \cdot v \cdot \int_{(2 B)^{c}} e^{i \lambda \Phi(x, y)} k_{\lambda}^{2}(x-y) \\
& \times \varphi(x, y) f(y) d y \\
:= & T_{\lambda}^{1} f_{2}(x)+T_{\lambda}^{2} f_{2}(x) .
\end{aligned}
$$

Let us first estimate $T_{\lambda}^{1} f_{2}(x)$. To do so, using Taylor's expansion and the compactness of $\operatorname{supp} \varphi$, we write

$$
\Phi(x, y)=\Phi(x, x)+P(x, y)+r_{N}(x, y)
$$

for $(x, y) \in \operatorname{supp} \varphi$, where $P(x, y)$ is a polynomial with $\operatorname{deg}$ $P<N$ and $\left|r_{N}(x, y)\right| \leq C|x-y|^{N}$ with $C$ independent of $x$ and $y$. Define

$$
R f(x)=p \cdot v \cdot \int_{(2 B)^{c}} e^{i \lambda P(x, y)} k_{\lambda}^{1}(x-y) \varphi(x, y) f(y) d y .
$$

Therefore

$$
\begin{aligned}
& e^{-i \lambda \Phi(x, x)} T_{\lambda}^{1} f_{2}(x)-R f(x) \\
& =\int_{|x-y| \leq 2 \lambda^{-1 / N}} e^{i \lambda P(x, y)}\left[e^{i \lambda r_{N}(x, y)}-1\right] \\
& \quad \times k_{\lambda}^{1}(x-y) \varphi(x, y) f(y) d y \\
& =\sum_{j=0}^{\infty} \int_{2^{-j} \lambda^{-1 / N}<|x-y| \leq 2^{-j+1} \lambda^{-1 / N}} e^{i \lambda P(x, y)}\left[e^{i \lambda r_{N}(x, y)}-1\right] \\
& \quad \times k_{\lambda}^{1}(x-y) \varphi(x, y) f(y) d y \\
& \equiv \sum_{j=0}^{\infty} T_{\lambda, j}^{1} f_{2}(x) .
\end{aligned}
$$


On $T_{\lambda, j}^{1} f_{2}(x)$, by the properties of $r_{N}$ and $k$, we have

$$
\left|T_{\lambda, j}^{1} f_{2}(x)\right| \leq C 2^{-j N} M f(x)
$$

So we have

$$
\left|T_{\lambda}^{1} f_{2}(x)\right| \leq C \sum_{j=0}^{\infty} 2^{-j N}|M f(x)|+C|R f(x)| .
$$

By Theorem A and Lemma 9, we have

$$
\left\|T_{\lambda}^{1} f_{2}\right\|_{L^{p, \kappa}(\omega)} \leq C\|f\|_{L^{p, \kappa}(\omega)} .
$$

Now, let us turn to estimate $T_{\lambda}^{2} f_{2}(x)$. We consider the following two cases.

Case $1(\lambda \leq 1)$. Similar to that estimate of $T_{\lambda}^{2}$ in Lemma 6 in [2], we have

$$
\left|T_{\lambda}^{2} f_{2}(x)\right| \leq C M(f)(x) .
$$

By Lemma 9 we have

$$
\left\|T_{\lambda}^{2} f_{2}\right\|_{L^{p, \kappa}(\omega)} \leq C\|f\|_{L^{p, \kappa}(\omega)} .
$$

Case $2(\lambda>1)$. We choose $\varphi_{0} \in C_{0}^{\infty}\left(\mathbb{R}^{n}\right)$ such that

$$
\begin{gathered}
\operatorname{supp} \varphi_{0} \subseteq\left\{x \in \mathbb{R}^{n}: 1<|x| \leq 2\right\}, \\
\phi_{2}(x)=\sum_{j=0}^{\infty} \varphi_{0}\left(2^{-j} x\right) .
\end{gathered}
$$

Let

$$
k_{\lambda, j}^{2}(x)=k(x) \varphi_{0}\left(2^{-j} \lambda^{1 / N} x\right)
$$

Then

$$
\begin{gathered}
T_{\lambda}^{2} f_{2}(x)=\int_{(2 B)^{c}} e^{i \lambda \Phi(x, y)} k_{\lambda}^{2}(x-y) \varphi(x, y) f(y) d y \\
\sum_{j=0}^{\infty} \int_{(2 B)^{c}} e^{i \lambda \Phi(x, y)} k_{\lambda, j}^{2}(x-y) \varphi(x, y) f(y) d y \\
\equiv \sum_{j=0}^{\infty} T_{\lambda, j}^{2} f_{2}(x) .
\end{gathered}
$$

For $T_{\lambda, j}^{2}$, by its definition, we can get

$$
\begin{aligned}
\left|T_{\lambda, j}^{2} f_{2}(x)\right| \leq C & \int_{2^{j} \lambda^{-1 / N}<|x-y| \leq 2^{j+1} \lambda^{-1 / N}} \\
& \times c \frac{1}{|x-y|^{n}}|f(y)| d y \leq C M(f)(x) .
\end{aligned}
$$

The inequality (36) also can be seen in [2]; we omit the details here.

By Lemma 9, we have

$$
\left\|T_{\lambda}^{2} f_{2}\right\|_{L^{p, \kappa}(\omega)} \leq C\|f\|_{L^{p, \kappa}(\omega)} .
$$

Therefore

$$
I_{2} \leq C\|f\|_{L^{p, \kappa}(\omega)}^{p} .
$$

This finishes the proof of Theorem 1 .
Proof of Theorem 2. It is sufficient to prove that there exists a constant $C>0$ such that

$$
\frac{1}{\omega(B)^{\kappa}} \int_{B}\left|T_{\lambda}^{*} f(x)\right|^{p} \omega(x) d x \leq C\|f\|_{L^{p, \kappa}(\omega)}^{p} .
$$

Fix a ball $B=B\left(x_{0}, r_{B}\right)$ and decompose $f=f_{1}+f_{2}$, with $f_{1}=f \chi_{2 B}$. Then we have

$$
\begin{aligned}
\frac{1}{\omega(B)^{\kappa}} \int_{B}\left|T_{\lambda}^{*} f(x)\right|^{p} \omega(x) d x & \\
\leq & C\left\{\frac{1}{\omega(B)^{\kappa}} \int_{B}\left|T_{\lambda}^{*} f_{1}(x)\right|^{p} \omega(x) d x\right. \\
& \left.+\frac{1}{\omega(B)^{\kappa}} \int_{B}\left|T_{\lambda}^{*} f_{2}(x)\right|^{p} \omega(x) d x\right\} \\
= & C\left\{J_{1}+J_{2}\right\} .
\end{aligned}
$$

Using Lemmas 3 and 7, we get

$$
\begin{aligned}
J_{1} & \leq C \frac{1}{\omega(B)^{\kappa}} \int_{2 B}|f(x)|^{p} \omega(x) d x \\
& \leq C\|f\|_{L^{p, \kappa}(\omega)}^{p} \cdot \frac{\omega(2 B)^{\kappa}}{\omega(B)^{\kappa}} \\
& \leq C\|f\|_{L^{p, \kappa}(\omega)}^{p} .
\end{aligned}
$$

We now estimate $J_{2}$.

For each $m \in \mathbb{N}$ and $j=1, \ldots, g_{m}$, we get

$$
a_{j m}(x)=\int_{\Sigma} \Omega(x, z) Y_{j m}(z) d \sigma_{z},
$$

where $\Omega(x, z)=|z|^{n} k(x, z)$. Then for a.e. $x \in \mathbb{R}^{n}$,

$$
\Omega(x, z)=\sum_{m=1}^{\infty} \sum_{j=1}^{g_{m}} a_{j m}(x) Y_{j m}\left(z^{\prime}\right),
$$

where $z^{\prime}=z /|z|$ for any $z \in \mathbb{R}^{n} \backslash\{0\}$. By Lemma 10, we have that, for any $x \in \mathbb{R}^{n}$,

$$
\begin{aligned}
\left|a_{j m}(x)\right| & =m^{-n}(m+n-2)^{-n}\left|\int_{\Sigma} \Omega(x, z) \Lambda^{n} Y_{j m}(z) d \sigma_{z}\right| \\
& =m^{-n}(m+n-2)^{-n}\left|\int_{\Sigma} \Lambda^{n} \Omega(x, z) Y_{j m}(z) d \sigma_{z}\right| \\
& \leq C(n) A m^{-2 n} .
\end{aligned}
$$

By Lemma 10 again, we can verify that, for any $\epsilon>0, N \epsilon$ $\mathbb{N}$, and a.e. $x \in \mathbb{R}^{n}$, if $|y-x| \geq \epsilon$, then

$$
\begin{aligned}
& \left|\sum_{m=1}^{N} \sum_{j=1}^{g_{m}} e^{i \lambda \Phi(x, y)} \frac{a_{j m}(x) Y_{j m}\left((x-y)^{\prime}\right)}{|x-y|^{n}} \varphi(x, y) f_{2}(y)\right| \\
& \quad \leq C(\epsilon) A\left|f_{2}(y)\right| .
\end{aligned}
$$


Therefore, from (43), (45), and the Lebesgue dominated convergence theorem, it follows that

$$
\begin{gathered}
T_{\lambda}^{*} f_{2}(x) \\
=\lim _{\epsilon \rightarrow 0} \int_{|x-y| \geq \epsilon} e^{i \lambda \Phi(x, y)} k(x, x-y) \varphi(x, y) f_{2}(y) d y \\
=\lim _{\epsilon \rightarrow 0} \sum_{m=1}^{\infty} \sum_{j=1}^{g_{m}} \int_{|x-y| \geq \epsilon} e^{i \lambda \Phi(x, y)} \frac{a_{j m}(x) Y_{j m}\left((x-y)^{\prime}\right)}{|x-y|^{n}} \\
\times \varphi(x, y) f_{2}(y) d y \\
=\lim _{\epsilon \rightarrow 0} \sum_{m=1}^{\infty} \sum_{j=1}^{g_{m}} a_{j m}(x) \int_{|x-y| \geq \epsilon} e^{i \lambda \Phi(x, y)} \frac{Y_{j m}\left((x-y)^{\prime}\right)}{|x-y|^{n}} \\
\times \varphi(x, y) f_{2}(y) d y .
\end{gathered}
$$

We write

$$
\begin{aligned}
& R_{j m} f_{2}(x) \\
& \quad=\int_{|x-y| \geq \epsilon} e^{i \lambda \Phi(x, y)} \frac{Y_{j m}\left((x-y)^{\prime}\right)}{|x-y|^{n}} \varphi(x, y) f_{2}(y) d y .
\end{aligned}
$$

It is easy to see that $R_{j m} f_{2}(x)$ is the oscillatory integral operator defined by (1). By Theorem 1 we have that $R_{j m}$ is bounded on weighted Morrey spaces. Therefore, by (44) and the above discussion we have

$$
J_{2} \leq C\|f\|_{L^{p, \kappa}(\omega)}^{p} .
$$

This finishes the proof of Theorem 2.

\section{Acknowledgments}

This work is supported by the National Natural Science Foundation of China (Grant no. 11001001), Natural Science Foundation from the Education Department of Anhui Province (nos. KJ2012B166, KJ2013A235).

\section{References}

[1] Y. Pan, "Uniform estimates for oscillatory integral operators," Journal of Functional Analysis, vol. 100, no. 1, pp. 207-220, 1991.

[2] S. Lu, D. Yang, and Z. Zhou, "On local oscillatory integrals with variable Calderón-Zygmund kernels," Integral Equations and Operator Theory, vol. 33, no. 4, pp. 456-470, 1999.

[3] C. B. Morrey, Jr., "On the solutions of quasi-linear elliptic partial differential equations," Transactions of the American Mathematical Society, vol. 43, no. 1, pp. 126-166, 1938.

[4] Y. Komori and S. Shirai, "Weighted Morrey spaces and a singular integral operator," Mathematische Nachrichten, vol. 282, no. 2, pp. 219-231, 2009.

[5] F. Chiarenza and M. Frasca, "Morrey spaces and HardyLittlewood maximal function," Rendiconti di Matematica e delle sue Applicazioni, vol. 7, no. 3-4, pp. 273-279, 1987.
[6] J. Peetre, "On the theory of $L^{p, \lambda}$ spaces," Journal of Functional Analysis, vol. 4, no. 1, pp. 71-87, 1969.

[7] S. Z. Lu, Y. Ding, and D. Y. Yan, Singular Integrals and Related Topics, World Scientific Publishing, River Edge, NJ, USA, 2007.

[8] E. Nakai, "Hardy-Littlewood maximal operator, singular integral operators and the Riesz potentials on generalized Morrey spaces," Mathematische Nachrichten, vol. 166, pp. 95-103, 1994.

[9] Y. Sawano and H. Tanaka, "Morrey spaces for non-doubling measures," Acta Mathematica Sinica, vol. 21, no. 6, pp. 15351544,2005

[10] H. Wang and H. P. Liu, "Some estimates for Bochner-Riesz operators on the weighted Morrey spaces," Acta Mathematica Sinica, vol. 55, no. 3, pp. 551-560, 2012.

[11] H. Wang and H. P. Liu, "Weak type estimates of intrinsic square functions on the weighted Hardy spaces," Archiv der Mathematik, vol. 97, no. 1, pp. 49-59, 2011.

[12] R. Ch. Mustafayev, "On boundedness of sublinear operators in weighted Morrey spaces," Azerbaijan Journal of Mathematics, vol. 2, no. 1, pp. 66-79, 2012.

[13] X. F. Ye and X. S. Zhu, "Estimates of singular integrals and multilinear commutators in weighted Morrey spaces," Journal of Inequalities and Applications, vol. 2012, article 302, 2012.

[14] H. Wang, "The boundedness of some operators with rough kernel on the weighted Morrey spaces," Acta Mathematica Sinica, Chinese Series, vol. 55, no. 4, pp. 589-600, 2012 (Chinese).

[15] H. Wang, "The boundedness of fractional integral operators with rough kernels on the weighted Morrey spaces," Acta Mathematica Sinica, Chinese Series, vol. 56, no. 2, pp. 175-186, 2013 (Chinese).

[16] S. He, "The boundedness of some multilinear operator with rough kernel on the weighted Morrey spaces," submitted, http://arxiv.org/abs/1111.5463.

[17] H. Wang, "Intrinsic square functions on the weighted Morrey spaces," Journal of Mathematical Analysis and Applications, vol. 396, no. 1, pp. 302-314, 2012.

[18] S. G. Shi, Z. W. Fu, and S. Z. Lu, "Boundedness of oscillatory integral operators and their commutators on weighted Morrey spaces," Scientia Sinica Mathematica, vol. 43, pp. 147-158, 2013 (Chinese).

[19] B. Muckenhoupt, "Weighted norm inequalities for the Hardy maximal function," Transactions of the American Mathematical Society, vol. 165, pp. 207-226, 1972.

[20] J. García-Cuerva and J. L. Rubio de Francia, Weighted Norm Inequalities and Related Topics, vol. 116 of North-Holland Mathematics Studies, North-Holland, Amsterdam, The Netherlands, 1985.

[21] R. F. Gundy and R. L. Wheeden, "Weighted integral inequalities for the nontangential maximal function, Lusin area integral, and Walsh-Paley series," Studia Mathematica, vol. 49, pp. 107124, 1974.

[22] E. M. Stein and G. Weiss, Introduction to Fourier Analysis on Euclidean Spaces, Princeton University Press, Princeton, NJ, USA, 1971. 


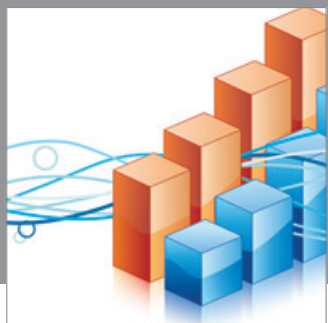

Advances in

Operations Research

mansans

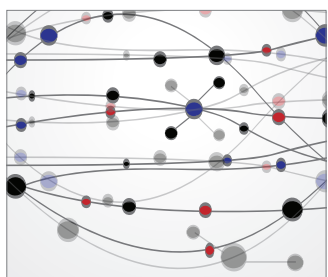

The Scientific World Journal
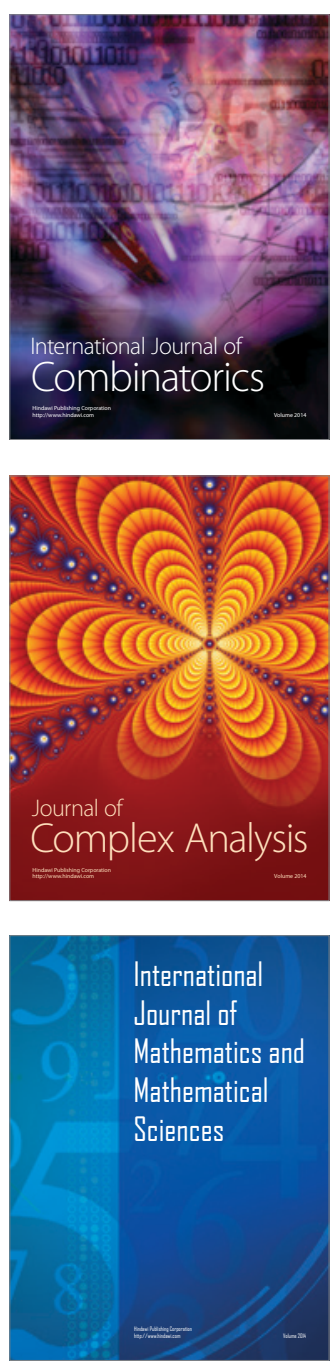
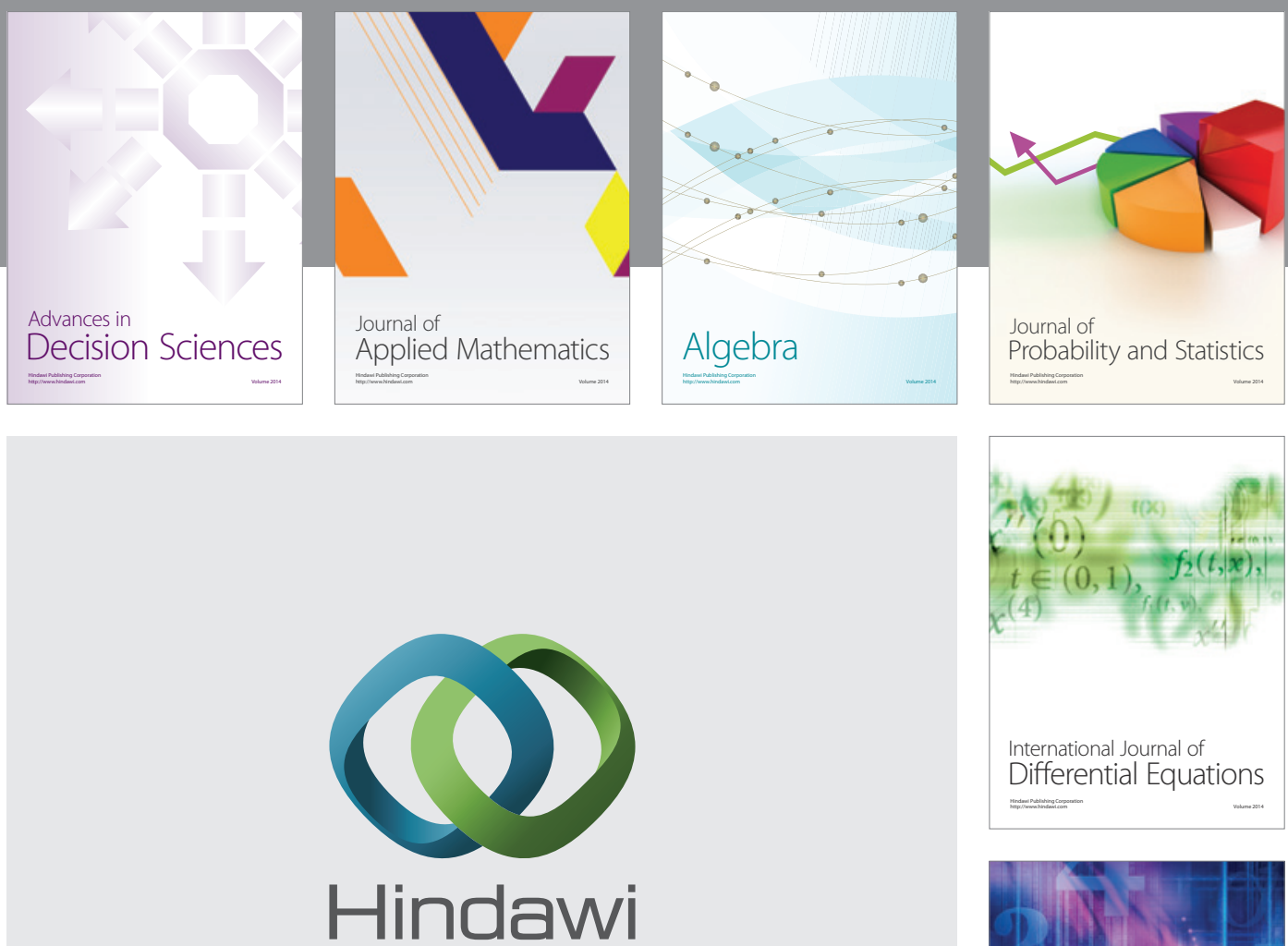

Submit your manuscripts at http://www.hindawi.com
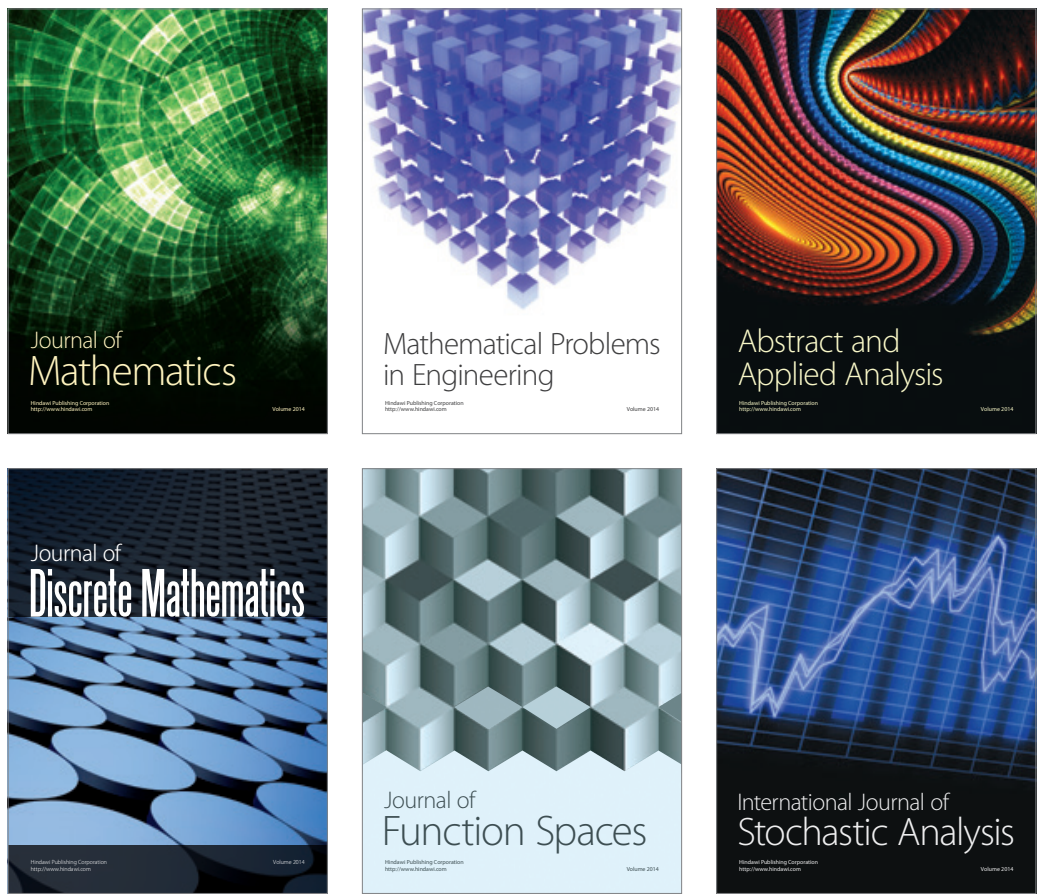

Journal of

Function Spaces

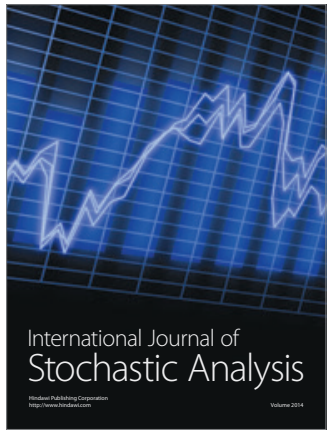

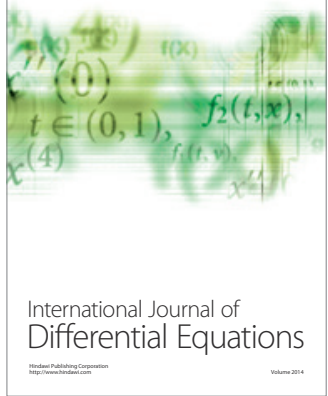
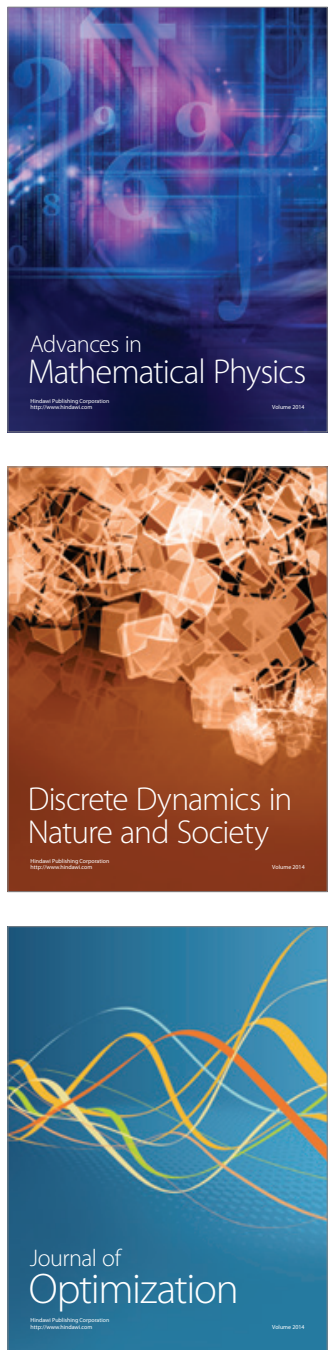